\title{
PLENARY SESSION
}

\section{PLENARY SESSION-I}

\section{PET/CT in Oncology Does it Really Help for Oncologic Patients of Bangladesh?}

\section{Abdul Wahab \\ Consultant \& Head of Department of Nuclear \\ Medicine, United Hospital Ltd., Gulshan, Dhaka.}

PET/CT is the most advanced fusion molecular imaging modality of Nuclear Medicine having its unique advantages of both anatomical and physiological information in a single study. More than $90 \%$ of all PET/ CT study is done by ${ }_{18} \mathrm{FDG}$ for oncologic patients for its diagnosis, staging and response to therapy and follow up in the whole world. Since the inception of PET/CT in United Hospital for the rst time in Bangladesh almost all study done for oncologic patients having experiences of shortening the diagnostic time, helping accurate diagnosis, proper staging for planning treatment, monitoring response to therapy and follow up after therapy for restaging resulting to overall cost $\mathrm{e}$ ectiveness of cancer treated patients even up to less than $30 \%$ compared to those who did not have undergone through PET-CT facilities'. So although PET CT is initially a costly modality for oncologic patients in perspective of Bangladesh still very much useful in Bangladesh with less su erings to patients, better survival rate, avoidance of unnecessary surgery or underuse/ overuse of chemotherapy misuse of radiotherapy and thus making it cost e ective \& gaining popularity day by day to doctors and patients still we have to go far away to make a regular application for oncologic patients.

KEYNOTE: PET/ CT -Positron emission tomography, FDG- uorodeoxy glucose

\section{Challenges of Hypothyroid Management- An Update}

\section{Prof. Dr. Faridul Alam}

\section{Professor and Director, BIHS Hospital and its Urban components}

Recent advances in our understanding of thyroid physiology may shed light on why some patients feel unwell while taking levothyroxine monotherapy. The purpose of this presentation to review the goals of levothyroxine therapy, the optimal prescription of conventional levothyroxine therapy, the sources of dissatisfaction with levothyroxine therapy, the evidence on treatment alternatives, and the relevant knowledge gaps. This presentation is intended to inform clinical decision-making on thyroid hormone replacement therapy; it is not a replacement for individualized clinical judgment. The issue of combined treatment with LT4 - LT3, other issue and procedure of LT4 treatment in line of the guidelines of ATA and other society's documents are highlighted to minimize the conflict. This presentation would be an attention to the basic science and its translational into thyroid hormone therapies, extensive consideration of ethical issues, focus on treatment itself, as opposed to other aspects of diagnosis, management and focus on evaluation of treatment alternatives. It is hoped to explore promising preclinical data for potential future therapies. For each topic, it is evaluated the scientific validity of the studies cited. It is expected this presentation will help clinicians providing care to patients with hypothyroidism. It is outlined what we believe in rational and optimal medical practice based on our evaluation of the evidence. However, areas of uncertainty and difference of opinion among experts remain. These recommendations are intended to inform the clinical decision-making 
process. For further study some references are given below.

\section{REFERENCES}

1. Garber JR, Cobin RH, Gharib H, Hennessey JV, Klein I,Mechanick JI, Pessah-Pollack R, Singer PA, Woeber KA,American Association Of Clinical E, American Thyroid Association Taskforce On Hypothyroidism In A 2012 Clinical practice guidelines for hypothyroidism in adults: cosponsored by the American Association of Clinical Endocrinologists and the American Thyroid Association. Thyroid 22:1200-1235.

2. Sullivan S, Wiersinga W2011 Guidelines of the American Thyroid Association for the diagnosis and management of thyroid disease during pregnancy and postpartum. Thyroid 21:1081-1125

3. Ain KB, Pucino F, Shiver TM, Banks SM 1993 Thyroid hormone levels a ected by time of blood sampling in thyroxine-treated patients. Thyroid 3:8-85.

4. De Groot L, Abalovich M, Alexander EK, Amino N, Barbour L, Cobin RH, Eastman CJ, Lazarus JH, Luton D,

5. Mandel SJ, Mestman J, Rovet J, Sullivan S 2012 Management of thyroid dysfunction during pregnancy and postpartum: an Endocrine Society clinical practice guideline. J Clin Endocrinol Metab 97:2543-2565

6. Wiersinga W, Duntas LH, Fadeyev VV, Nygaard B, Vanderpump MPJ 20122012 ETA Guidelines: the use of L-T4 + L-T3 in the treatment of hypothyroidism. Eur Thyroid J 1:55-71.

7. Raverman LE, Ingbar SH, Sterling K 1970 Conversion ofthyroxine (T4) to triiodothyronine (T3) in athyreotic human subjects. J Clin Invest 49:855-864

8. Oppenheimer JH, Schwartz HL, Surks MI 1972 Propylthiouracil inhibits the conversion of L-thyroxine to L-triiodothyronine. An explanation of the antithyroxine e ect of propylthiouracil and evidence supporting the concept that triiodothyronine is the active thyroid hormone. J Clin Invest 51:2493-2497.

9. Razvi S, Weaver JU, Butler TJ, Pearce SH 2012 Levothyroxine treatment of subclinical hypothyroidism, fatal and nonfatal cardiovascular events, and mortality. Arch Intern Med 172:811-817.

10. Jonklaas J, Davidson B, Bhagat S, Soldin SJ 2008 Triiodothyronine levels in athyreotic individuals during levothyroxine therapy. JAMA 299:769-777.

11. Escalante DA, Arem N, Arem R 1995 ;Assessment of interchangeability of two brands of levothyroxine preparations with a third-generation TSH assay. Am J Med 98:374-378.

12. Bach-Huynh TG, Nayak B, Loh J, Soldin S, Jonklaas J 2009 Timing of levothyroxine dministration a ects serum thyrotropin concentration. J Clin Endocrinol Metab 94:3905-3912. 129.

13. 12. Bolk N, Visser TJ, Nijman J, Jongste IJ, Tijssen JG,
Berghout A 2010 E ects of evening vs morning levothyroxine intake: a randomized double-blind crossover trial. Arch Intern Med 170:1996-2003

14. Serter R, Demirbas B, Korukluoglu B, Culha C, Cakal E, Aral Y 2004 The e ect of L-thyroxine replacement therapy, on lipid based cardiovascular risk in subclinical hypothyroidism. J Endocrinol Invest 27:897-903

15. Turner MR, Camacho X, Fischer HD, Austin PC, Anderson GM, Rochon PA, Lipscombe LL 2011 Levothyroxine dose and risk of fractures in older adults: nested case-control study. BMJ 342:d2238

16. Bugdaci MS, Zuhur SS, Sokmen M, Toksoy B, Bayraktar, B, Altuntas Y 2011 The role of Helicobacter pylori in patients with ypothyroidism in whom could not be achieved normal thyrotrophic levels despite treatment with high doses of thyroxin. Helicobacter 16:124-130

17. 1Curran PG, DeGroot LJ 1991 The e ect of hepatic enzymeinducing drugs on thyroid hormones and the thyroid gland. Endocr Rev 12:135-150.

18. Kaptein EM, Beale E, Chan LS 2009 Thyroid hormone therapy for obesity and nonthyroidal illnesses: a systematic review. J Clin Endocrinol Metab 94:3663-3675

19. Taylor J, Williams BO, Frater J, Stott DJ, Connell J 1994 Twice-weekly dosing for thyroxine replacement in elderly patients with primary hypothyroidism. J Int Med Res 22:273-277.

20. Ott J, Promberger R, Kober F, Neuhold N, Tea M, Huber JC, Hermann M 2011 Hashimoto's thyroiditis a ects symptom load and quality of life unrelated to hypothyroidism: a prospective case-control study in women undergoing thyroidectomy for benign goiter. Thyroid 21:161-167. 417. Pop VJ, Maartens LH, Leusink G, van Son MJ, Kn

\section{Essential Regulatory Requirements For Establishing PET-CT Facility In Nuclear Medicine Practice of Bangladesh}

1Shahana Afroz, 2Raihan Hussain R \& 3Meherun Nahar

\author{
${ }_{1}$ Prof. Dr. Shahana Afroz, Member, Bangladesh \\ Atomic Energy Regulatory Authority (BAERA)
}

${ }_{2}$ Prof. Dr. Raihan Hussain Chief Medical O cer, Natinal Institute of Nuclear Medicine and Allied Sciences (NINMAS).

${ }_{3}$ Dr. Meherun Nahar, Principal Scientific Officer, BAERA

\section{Introduction}

Noninvasive imaging done in Nuclear Medicine $(\mathrm{NM})$ is of fundamental and increasing importance 
Over the past few decades positron emission tomography (PET) and PET/CT (computed tomography) have revolutionized the care of cancer patients in developed countries and are increasingly being adopted in emerging economies.

PET has been, and still is, one of the fastest growing fields in medical imaging. There are several reasons for the rapid development of this imaging technology. The ability to provide, in a single imaging session, detailed anatomical and metabolic/functional information, which has a powerful synergistic effect that is greater than the sum of the two individual techniques, has established $\mathrm{PET} / \mathrm{CT}$ as an indispensable imaging procedure in the management of many different types of cancer. The quality and reliability of the images acquired on a PET/CT scanner depend on the quality of the imaging technique. Proper cancer management requires highly accurate imaging to characterize, stage, restage, assess response to therapy, prognosticate and detect recurrence. Such information is critical in a disease that often requires the correct initial treatment in order to improve the chance of successfully curing the patient.

Presently, every well-established hospital would like to procure Medical Cyclotron and positron emission tomography-compound tomography (PET-CT) facility in their NM department. Because cyclotronproduced radionuclides have higher energy than the other routinely used radionuclides for diagnosis, it becomes essential for user to know about the regulatory requirement and radiation safely precautions that one has to take for the installation of this new modality in their premises.

Peaceful uses of ionizing radiation are wide spread and virtually indispensible to the modern society. In addition to the many benefits afforded by the beneficial uses of radiation, there are also associated hazards. Therefore it is essential for every country to have adequate infrastructures to ensure the safety and security of all radiation sources. Bangladesh
Atomic Energy Commission (BAEC) constituted "The Nuclear Safety and Radiation Protection Division" in 1987, later renamed as "The Nuclear Safety and Radiation Control Division (NSRCD)". Eventually an independent regulatory authority was established in 2013 and named as Bangladesh Atomic Energy Regulatory Authority (BAERA). Legal Instruments of BAERA includes i) Nuclear Safety and Radiation Control (NSRC) Rules 1997 ii) Bangladesh Atomic Energy Regulatory ( BAER ) Act- 2012 and various regulatory Guides through which BAERA regulates use of radiations in health, agriculture, industry and so on.

The various stages of approval of PET-CT facility by the Regulatory Authority and important steps that one has to know/follow before planning for this new facility are summarized in the following sections.

\section{Site and lay out plan approval}

The user has to submit to the competent regulatory authority the proposed layout plan, site plan, and elevation drawing of the facility indicating the poor, nature of occupancy around, above and below for necessary approval.

\section{Submission of regulatory consent form}

The user has to submit the details of the completion of the construction work as per the approved plan, installation of equipment, procurement of radiologic protection accessories, enrollment of radiation workers in Personal Monitoring Service and availability of qualified staffs per NSRC Rule 1997

\section{Precommissioning inspection}

In this stage, the pre commissioning inspection of the facility will be carried out by BAERA official(s) to ensure that the construction of NM facility is as per the approved plan.

\section{Approval for commissioning/routine operation}

On ensuring the compliance of the requirement as specified in NSRC rule 1997 for the safe handling of 
radioactive material in the approved NM facility, the authorization for the procurement of radioactive material indigenously or no objection certificate (NOC) for procurement of radioactive material from abroad will be issued for the stipulated time period by BAERA.

\section{Work practice}

In NM facility, the radiopharmaceutic formulation should be prepared, handled, administered to the patients, and disposed of in a safe manner taking into account adequate radiation protection measures. Radioisotopes should be stored, used, and transported safely and securely all the time.

\section{Waste disposal}

In Bangladesh, radioactive waste management is governed by Atomic Energy Regulatory Authority Chairman, BAERA is the competent authority. Implementation of the rules is primarily ensure safety of the public and the environment.

\section{Decommissioning}

When the NM facility is no longer to be used, the permission for decommissioning should be obtained from the BAERA

\section{Radiation protection aspects of PET-CT facilities}

NM facility with PET-CT employs relatively large activities of high-energy photon emitting radioisotopes. This coupled with the current dose limits for members of the public, can result in a shielding requirement. Even modest reductions in the radiation levels at $511 \mathrm{keV}$ require significant amounts of shielding. A throughout and site-specific evaluation has to be made for each facility according to BAERA act.

\section{Shielding calculations}

Presently, Radiation Control Division (RCD) task group are responsible for PET and PET-CT shielding requirements are being used for carrying out the shielding calculations.

\section{Radiation safety issues}

Vendors are providing specialized equipment like automated dose dispenser, dose calibrator, well counter etc to reduce exposure to operation personnel in the PET-CT facility and to improve instrument performance in the higher radiation background found in the lot of laboratories which has to be ensured.

\section{Staff requirement for nuclear medicine laboratory}

A facility to be approved by the BAERA should the adequate stu /personnel including Nuclear Medicine Physicians, Medical Physicist, Radio chemist, Nuclear Medicine Technologists and Radiation Control Officer etc. Everyone should have a solid training and background knowledge about radioisotope handling and administration.

The PET/CT written report is the primary mode of communication between the physician interpreting an imaging study and the referring physician. Generating a high quality PET/CT report is perhaps more challenging than generating a report for other imaging studies because of the complexity of this hybrid imaging modality. So the persons who are reporting must have in depth knowledge and expertise in Nuclear Medicine. This can be achieved by having post graduate academic degree in Nuclear Medicine or having a long professional training/ experience in Nuclear Medicine. More over they should have additional experience in PET/CT.

\section{Operating suggestion}

Transporting, positioning the patient and detail instruction for patients should be completed according to the regulation of BAERA to minimize the radiation exposure to the technologists.

In addition to the above mentioned areas i) Personnel monitoring services for staff members ii) Radiation monitoring devices and iii) Radiation safety devices has to be carefully evaluated and ensured according to the NSRC rule 1997 of BAERA.

\section{Conclusion}

PET-CT facilities present somewhat different design requirements than conventional nuclear medicine departments and are more likely to require additional radiation shielding. By use of appropriate design and by maintaining good operating practices, radiation doses to staff, public and 
environment can be kept to acceptable limits.

\section{References}

1. Nuclear Safety and Radiation Control (NSRC) Rules -1997

2. Bangladesh Atomic Energy Regulatory (BAER) Act-2012

3. Codes and Guides- Atomic Energy Regulatory Board (AERB), India.

4. Pankaj Tandon, "Regulatory requirement for designing PET-CT facility in India" Indian J Nucl.Med. 2010;25(2):39-43

5. Patient Radiation Protection ManualFor facilities using medical ionizing radiation (FIRST EDITION August 2013) Medical Exposure Radiation Unit, IAEA

6. Ryan D et al," Reporting Guidance for Oncologic ${ }_{18} \mathrm{~F}-\mathrm{FDG}$ PET/CT Imaging" Journal of Nuclear Medicine. 2013 May; 54 (5)

\section{Targeted Alpha Therapy: The Next Frontier in Nuclear Medicine Therapeutics}

Lutfun Nisa MBBS, M Phil. Kamila Afroj Quadir, P hD, Shamim MF Begum MBBS, DNM, Raihan Hussain MBBS, PhD, Mizanul Hasan MBBS, M Phil.

\section{National Institute of Nuclear Medicine \&}

Allied Science (NINMAS), Dhaka.

Background: Targeted alpha therapy (TAT) is a new experimental, systemic therapy that targets cancer cells and tumor capillary endothelial cells by intravenous injection of an alpha immunoconjugate (AIC). The AIC is formed by labeling the cancer targeting monoclonal antibody with the alpha emitting radioisotope Bi-213 using a bifunctional chelator. The monoclonal antibody is raised against antigens that are over expressed by cancer cells. One such antigen is the MUC1 antigen. There are several centers actively involved in TAT, notably in Europe, the US and Australia. Bangladesh is considering a proposal for Clinical Phase 1 trial in TAT and is in the process of obtaining the required ethical clearance. The proposed TAT technique in Bangladesh will be discussed and highlighted in this presentation.

Objective: The aim of the presentation is to project the potentiality of TAT technology as a safe therapeutic option in patients with advanced MUC1 positive cancers.

Method: The monoclonal antibody C595 against MUC1 and the Actinium:Bismuth generator required for the study will be obtained from Australia. Patients with stage four MUC1 positive cancers having progressive disease and those who have either completed or have declined other systemic therapies will be included in the trial. Cohorts of 3 subjects with end-stage cancer will be treated with escalating doses of $5 \mathrm{mCi}$, then $10,15,20,25,30 \mathrm{mCi}$ every 2 months. If adverse events are seen in one patient then the maximum tolerance dose will be the preceding dose. Patients will be followed up for 12 months with emphasis on the detection of delayed radiation nephrosis.

Outcome: The proposed study will bring together the highly selective features of a unique targeting system with the high cytotoxicity of alpha particles for treatment of mucin (MUCI) expressing tumors of the breast, ovary, pancreas and prostate. It will optimize the key parameters of targeted alpha therapy, ie stability and specific activity of the alpha - conjugate and maximum tolerance dose that may lead to a much higher rate of tumor control.

Conclusion: Currently there is no systemic treatment that can inhibit the progression of cancer that leads invariably to the death of the patient. TAT has the potential for durable therapeutic responses in stage four cancer patients.

\section{Special lecture: Nuclear Medicine in Bangladesh - Through the ages}

Prof. Dr. Raihan Hussain, MBBS, DNM, MACR, FANMB, Ph D.

\section{National Institute of Nuclear Medicine and Allied Sciences, BAEC}

\begin{abstract}
Bangladesh has a long and rich history of nuclear medicine for over sixty years. The progress in science and technology is always challenging in a developing country. In 1958, work for the rst NM
\end{abstract}


facility was commenced in Dhaka in a tin-shed known as 'Radioisotope Centre' and was officially inaugurated in 1962. Since the late 50s of the last century nuclear medicine in Bangladesh has significantly progressed through the years in its course of development, but still the facilities are inadequate. At present there are 20 nuclear medicine establishments with 3 PET-CTs, 42 gamma camera/SPECTs having $95 \mathrm{NM}$ physicians, 20 physicists, 10 radiochemists and 150 technologists. The Society of Nuclear Medicine, Bangladesh (SNMB) was formed in 1993 and publishing its offcial journal since 1997. The nuclear medicine community also has close relationships with international organizations like IAEA, ARCCNM, AOFNMB, ASNM, WFNMB, WARMTH etc. The history and the present scenario of the status of nuclear medicine in Bangladesh are being described here.

Key Words: History, Nuclear Medicine, Bangladesh

\section{PROFFERED ORAL PRESENTATION}

\section{Abstract No. OP-1}

A Meta-analysis on Usefulness of the Maximum Standard Uptake Value (SUV max) measured by F-18-FDG PET in Patients with Uterine Cervical Cancer

1Azmal Kabir Sarker, ${ }_{2}$ Hyung-Jun Im, ${ }_{2}$ Gi Jeong Cheon, ${ }_{3}$ Hyun Hoon Chung, ${ }_{2}$ Keon Wook Kang, ${ }_{2}$ JuneKey Chung, 2Euishin Edmund Kim, 2Dong Soo Lee

${ }_{1}$ National Institute of Nuclear Medicine and Allied Sciences, Dhaka; 2 Department of Nuclear Medicine, Seoul National University Hospital, Korea; ${ }_{3}$ Department of Obstetrics and Gynecology, Seoul National University College of Medicine, Korea

\section{Abstract}

Purpose: This meta-analysis was conducted to evaluate the prognostic value of the maximum standard uptake value (SUVmax) measured in pretreatment primary lesions and metastatic lymph nodes (LN) on F-18- uorodeoxyglucose-positron emission tomography (F-18-FDG-PET) scans in patients with uterine cervical cancer.

Methods: In order to identify the studies that investigated association of SUVmax of primary tumor or metastatic pelvic (PLN) or para-aortic (PALN) lymph node with survival outcomes in uterine cervical cancer, a systematic search of EMBASE and MEDLINE was performed using keywords of "positron emission tomography (PET)," "uterine cervical cancer," and prognosis. Event-free survival (EFS) and overall survival (OS) were evaluated as outcomes. The impact of SUVmax on survival was measured by the effect size of the hazard ratio (HR).

Results: Fourteen eligible studies including 1150 patients were analyzed. Patients with a high SUVmax primary showed a worse prognosis with a HR of 2.66 (95\% CI, 1.90-3.74, $\mathrm{p}<0.00001$ ) for adverse events and a HR of 2.45 (95\% CI, 1.74-3.45, p < 0.00001) for death. Patients with high SUVmax in metastatic pelvic LN (PLN) showed a worse prognosis with a HR of 2.92 (95\% CI, 1.94-4.39, $\mathrm{p}<0.00001$ ) for adverse events and a HR of $2.66(95 \% \mathrm{CI}, 1.60-4.43, \mathrm{p}=0.0002)$ for SUVmax in PLN for death. In addition, high SUVmax in metastatic para-aortic LN (PALN) was associated with a worse prognosis, with a HR of 4.41 (95\% CI, $2.32-8.38, \mathrm{p}<0.00001$ ) for death.

Conclusions: SUVmax measured by of $18 \mathrm{~F}-\mathrm{FDG}$ PET are significant prognostic factors of outcome in patients with uterine cervical cancer. Patients with a high SUVmax primary, PLN and PALN are at higher risk for adverse event or death.

\section{Abstract No. OP-2}

\section{Management of Papillary Thyroid Carcinoma with Pulmonary Metastases- Success Story of INMAS Rajshahi}

1Kabiruzzaman Shah, ${ }_{2}$ Mostofa Shamim Ahsan, 1Nasrin Begum, 1 Mosharruf Hossain, 1 Parvez Ahmed, 1Shariful Islam Chowdhury

${ }^{1}$ Institute of Nuclear Medicine and Allied Sciences 
(INMAS), Rajshahi.

${ }^{2}$ National Institute of Nuclear Medicine and Allied Sciences (NINMAS), Dhaka.

Introduction: Thyroid cancer is the most common endocrine malignant tumor. Papillary thyroid carcinoma (PTC) comprises $80 \%$ of the all thyroid cancers and commonly metastasizes to regional lymph nodes. Distal metastasis is rare, occurs in 5\% of the cases and more frequently involved sites are lung and bone. In case PTC with distal metastasis, 10 years survival rate is approximately $25-40 \%$. The prognosis of the disease depends upon the appropriate treatment. The prognosis is excellent when given appropriately. The recurrence rate of DLC is relatively and accounts from $10-30 \%$ cases. Hence lifelong follow up is needed in all DTC for better outcome.

Here we reported two cases of PTC with pulmonary metastasis were successfully treated with radioiodine therapy at a peripheral center.

Case Reports: A 23 yrs old unmarried female patient with the diagnosis of PTC were referred to our institute in 2006 for radioiodine therapy after near thyroidectomy. Her base line study showed 60mIU/L (0.3-5.00 mIU/L) serum TSH level \& large dose scan (LDS) showed high small residual functioning tissue in thyroid bed. She treated with 138 mci of I-131. After that replacement therapy with thyroxin preparation started and follow up was done at regular interval up to 2009. During next four years the patient had her event free follow-up. But in year 2014 she developed respiratory problem and multiple patchy opacities were revealed on chest X-ray. Large dose whole body with I-131 scan showed uptake in the lungs and treated with 200mci I-131 and followed by LT4 therapy. She was strictly advice for regular follow up according to thyroid carcinoma management protocol. Her last follow-up WBS was done on June 2015, LDS was negative for metastasis.

A 20 Years old male patient was diagnosed as papillary carcinoma of thyroid with local nodal metastases in right cervical group of lymph nodes in Segment II, III and IV. He underwent total thyroidectomy with radical neck dissection and referred to us our institute for radio-iodine ablation in March 2014. All investigation was done according to our protocol including LDS. On baseline LDS, small residual tissue at thyroid bed was noted as well as iodine uptake was also present in upper part of right lung parenchyma. Clinically patient had respiratory symptoms and radiologically patchy opacity in upper zone of right lung. Our diagnosis was pulmonary metastasis. Radio-ablation was done April 2014 with 200 mci I-131 followed by replacement therapy. During treatment no complication was developed. He came for follow up after 3 months, during 1st visit all investigation were performed according to cancer thyroid.

Key Words: Papillary thyroid carcinoma (PTC), large dose whole body scan (LDS), Pulmonary metastasis.

\section{Abstract No. OP-3 \\ Simulation of motion induced data from real SPECT data}

1Md. Nahid Hossain, 2 Kamila Afroj Quadir, 3Adnan Kiber, ${ }_{4}$ Roger Fulton

${ }_{1}$ National Institute of Nuclear Medicine \& Allied Sciences, Dhaka

2Bio-Science Division, Bangladesh Atomic Energy Commission

${ }_{3}$ Department of Electrical \& Electronic Engineering, University of Dhaka

${ }_{4}$ Department of Medical Physics, Westmead Hospital, Australia

Introduction: In a Single Photon Emission Computed Tomography (SPECT) study, the data acquisition is performed over a relatively long time, typically in the range of 5-30 minutes. For longer 
period patient movement frequently occurs in clinical procedure. This movement causes misalignment of the projection frames, which degrades the image quality. In this work, different SPECT studies by using phantom without motion were acquired and by using algorithm, the motion induced data were simulated from these real data. When these simulated data were created, different techniques of motion estimation could be applied to correct the subject's motion.

Methods: Different data without motion were acquired by using a Trionix Triad triple-head SPECT camera and a Ho man 3D brain phantom. Simulated data were evaluated and Mean Square Difference (MSD) was calculated using two different developed algorithms respectively. Algorithm was developed for simulation of new data from the real motion free data. The both algorithm were created with Interactive Data Language (IDL) programme.

Results: The simulated data was produced by adding partial projection data with the aim to produce a new data set so that it simulates motion induced data. We calculated the MSD from the simulated data by analyzing each projection frames. These showed the significant MSD values between two different projection frames which were obtained from two different SPECT acquisitions. By using the simulated data, motion correction could be performed.

Discussion: Motion induced simulated data is useful in quantifying motion estimation and develop correction methods that will allow derivation of motion free data. Motion detection techniques are classifed into two categories, external based or internal based. The simulated data are used for internal based technique.

Conclusion: The eld of motion detection and correction in SPECT is very open to future novel ideas especially software based improvement of motion estimation, characterization and compensation. The simulated data is very essential for examining the algorithm base methods.

\section{Abstract No. OP-4 \\ Ultrasound Guided Ethanol Sclerotherapy in Benign cystic thyroid nodule}

Md. Mosharruf Hossain, Kabiruzzaman Shah, Nasrin Begum Laz and Parvez Ahmed.

Institute of Nuclear Medicine \& Allied Sciences, Rajshahi, Rajshahi Medical College Hospital Campus

Background: Benign cystic thyroid nodule is one of the common thyroid illnesses in Bangladesh either solitary or multiple. Benign cystic nodules are usually treated by surgery in our country which is risky, time consuming and costly. Ethyl alcohol (ethanol- 99.9\%) is a sclerosing agent which can be used under high resolution ultrasound guidance for the treatment of benign cystic thyroid nodule.

Objectives: To see the effectiveness and side effects of Ultrasound guided ethanol sclerotherapy and thyroid functional status after this therapy.

\section{Materials and methods:}

This study was done in a private center of Rajshahi named UTC Ultrasound \& Thyroid Center during the period of January 2012 to January 2014. A total of 6 patients were included for this treatment. Before treatment HRUS, T3, T4, TSH and FNAC were done in each patient. Only the purely cystic nodules were included for this treatment. Almost all the uids were aspirated from the nodules by $19 \mathrm{G}$ needle under HRUS guidance. Then ethanol was injected inside the nodule by the same needle without withdraw from the nodule. The amount of ethanol was injected within the nodule is about $1 / 2$ of the aspirated uid volume. After ethanol injection the needle was withdrawn from the nodule. About 10-15 minutes after ethanol injection we observed the patient in a low room temperature to see any side e ect.

\section{Results:}

The range of nodule uid volume was 12- $60 \mathrm{ml}$. After ethanol slerotherapy we have followed up all 
the patients by HRUS and T4, TSH at 1, 3 and 6 months of therapy. All the nodules were completely disappeared within 3-6 months of therapy. Mild burning sensation was seen in two patients. Only single therapy was sufficient for four patients except two whose uid volume of nodules were more than 50 $\mathrm{ml}$. Second dose of sclerotherapy was given in these two patients after 3 months of rst therapy and complete disappearance were seen after 3 month of 2 nd dose.

\section{Conclusion:}

Ultrasound guided ethanol sclerotherapy is a highly effective, very cheap and time saving modality for benign cystic thyroid nodule as well as minimally invasive and almost without any side effect. This modality can be a used for the treatment of benign purely cystic thyroid nodule as alternative to surgery.

\section{Abstract No. OP-5}

\section{Chemiluminescence Immunoassay: A non- isotopic alternative to ${ }_{25}$ l labeled immunoassay}

Mohammad Anwar-ul Azim, Mizanul Hasan,

Israque Hossain Ansari and Faria Nasreen

\section{National Institute of Nuclear Medicine and Allied Sciences, BAEC, BSMMU, Shahbagh, Dhaka.}

\section{Abstract}

Chemiluminescence immunoassay (CLIA) is a method to determine the concentration of samples according to the intensity of the luminescence that the chemical reaction emits. Direct chemiluminescent (CL) reactions involve the reaction in between a substrate and an oxidant to from a product or intermediate. Then some fraction of the product or intermediate will be formed in an electronically excited state, which can subsequently relax to the ground state with emission of a photon. The substrate is the CL, precursors, which is converted into the electronically excited molecule, responsible for light emission or acting as the energy as transfer donor in indirect CL reactions.
The most popular CL substrates are luminol, isoluminol and their derivatives, acridinium ester (AE) derivatives, peroxidase and alkaline phosphatase. The quantitative inhibition of binding of labelled antigen to antibody by increasing concentrations of unlabeled antigen forms the basis of radioimmunoassay (RIA). Immunoradiometric assay (IRMA) is an alternative procedure to RIA, which relies on the conversion of antigen to a labelled derivative by reaction with labeled antibody. The derivative is then measured directly. Both RIA and IRMA have relied on the use of ${ }_{125} 1$ as the label. This isotope is a moderately high-energy $\gamma$-emitter with a half-life of 60 days and its sensitivity of detection is excellent, approximately $5 \times 10_{-18} \mathrm{~mol}$. Chemiluminescence immunoassay o ers a viable and logical alternative to a range of analytical immunoassay based on the use of radioisotopes. First and foremost, they eliminate the use of radioactive labels and the related concerns regarding exposure of personnel to radiation and waste disposal. In addition, CLIA assays use reagents with a longer shelf life, a higher sensitivity, and a shorter running time. However, traditional radioimmunoassays need great consumption of time, reagents and labor, which limit their clinical applications. Due to its advantage in sensitivity, speed, high-throughput and automation, CLIA system has become one of the most important members in in vitro diagnosis (IVD) medical devices. Therefore, more and more medical experts and chemists are interested in CLIA. The development of CLIA is dependent on the application of the sensitive and selective chemiluminescent probe. A new chemiluminescence immunoassay system with AE technology will be installed in the in vitro division of National Institute of Nuclear Medicine and Allied Sciences (NINMAS). Parallel to the existing gold standard radioimmunoassay method, introduction of chemiluminescence immunoassay will de nitely improve the competency of in vitro laboratory of NINMAS in the detection and monitoring of tumour markers, thyroid and reproductive hormones. 
Early diagnosis can help to restore euthyroid state which will protect the patient from further paralytic attack. Here is a case report who presented with repeated episodes of paralysis cause by hypokalaemia associated with Thyrotoxicosis.

Case report: One 38 yrs old male in my rural area (Bashbaria, Rampal, Bagerhat) presented with weakness in both upper \& lower limbs and unable to walk in last April. He worked hard during the day and took dinner with his family, at $11 \mathrm{pm}$ he felt weakness and gradually it was worsening. I examined the patient and found diminished motor function with raised heart rate (90 b/minute-regular). He reported that for the last $7 \mathrm{yrs}$ he experienced such paralytic attack with an interval of 3-6 months and spontaneously recovered within 2-3 days. Moreover he noticed that green coconut water and oral saline were helpful for getting strength in muscles. His all investigations supported that he had been suffering from Thyrotoxicosis for the last $7 \mathrm{yrs}$ and took Tab Carbimazol 5mg ( 2 tab thrice daily but very irregularly) but the exact cause of paralysis could not be clearly explored. According to his parents statement rest of his three brothers and one sister also had been su ering from periodic limbs weakness but not so severe like him.

Keeping in mind the fear of respiratory muscles paralysis I referred the patient to Khulna Medical College at midnight. Serum electrolytes testing on admission revealed that his potassium level was mildly reduced (3.37 mEq/L, normal: 3.5-5 $\mathrm{mEq} / \mathrm{L}$ ); sodium and chloride levels were within normal. Regarding thyroid hormones: T3, T4 \& TSH were $1.04 \mathrm{nmol} / \mathrm{L}$, $187 \mathrm{nmol} / \mathrm{L} \& 0.15 \mathrm{mIU} / \mathrm{L}$ respectively. His ECG findings were normal. One medial board was formed for proper evaluation and after exclusion of all other possible causes of paralysis the patient was finally diagnosed as Thyrotoxic periodic paralysis. His Thyrotoxic features and hypokalaemic conditions were corrected with Tab Carbimazol
$5 \mathrm{mg}$ (3 tab thrice daily), Tab Spironolactone $250 \mathrm{mg}$ (1 tab at morning), Tab Acetazolamide $250 \mathrm{mg}$ (1 tab thrice daily). He was discharged from the hospital after 7 days. Genetic counseling was not possible. For prevention of such periodic paralysis same medicines were also recommended for his brothers \& only sister. Moreover they were advised to avoid large meal, hard working, cold \& hot weather simultaneously, and encouraged to take more banana and green coconut water.

Key Words: Thyrotoxicosis, Hypokalaemia, Periodic Paralysis

\section{Abstract No. OP-7 \\ Roles of Nuclear Medicine Professionals in Case of a Nuclear or Radiological Emergency in Bangladesh}

1A.S. Mollah, ${ }_{2}$ Aleya Begum, ${ }_{1}$ M. A. Hossain and 1A.Z.M. Salahuddin

${ }_{1}$ Department of Nuclear Science and Engineering

Military Institute of Science and Technology, Mirpur, Dhaka-1216.

2Bangladesh Atomic Energy Commission, Paramanu Bhaban, Agargaon, Dhaka-1207.

\section{Introduction}

Bangladesh has since long been using atomic energy in the elds of Medicine, Industry, Agriculture, Education and Research for peaceful purposes. The uses of different sources of ionizing radiation are increasing steadily in all the above mentioned elds for socio-economic development of the country. Establishment of a Nuclear Power Plant (NPP) is under active consideration of GOB for meeting the ever growing electricity crisis. It is anticipated that radiation hazards may happen in case of any incident or accident causing nuclear or 
Regulatory (BAER) Act-2012 and Nuclear Safety and Radiation Control (NSRC) Rules-1997, individual organization shall have a nuclear or radiological emergency response plan to meet any on-site and off-site radiological consequences. Radiological emergency preparedness has been gaining increased attention due to recent events such as the 2011 Fukushima Daiichi nuclear emergency, which revealed a shortage of experts in the eld of radiation and radionuclide detection (1). The main objective of this paper is to describe the following points:

a) Key elements of proposed national nuclear or radiological response plan

b) Roles of nuclear medicine professionals in case of a nuclear or radiological emergency

\section{Materials and Methods}

As per NSRC Rules-97, a national nuclear or radiological emergency response (NNRER) plan was prepared in 2005 and submitted to BAEC for approval (2). The proposed NNRER plan was revised several times and submitted to IAEA by BAEC in 2014 for further improvements. BAEC has established a national institute in Dhaka along with 15 other Institute of Nuclear Medicine (INM) spread all over the country. In general, the major facilities are available in different INM: scintillation gamma camera, well counter, Geiger counter, thyroid probe, portable $\mathrm{Pb}$-shield, $\mathrm{Pb}$-apron, etc. These facilities can be used during radiological emergency. Others nuclear equipment such as portable radionuclide identi er, multichannel analyzer, whole body counter, contamination monitor, neutron monitor, air sampler, smear paper, radiation caution symbols etc. can be obtained from di erent Health Physics Laboratories of BAEC.

\section{Discussions}

Many quali ed nuclear medicine professionals (NMPs), such as, nuclear medicine physician, medical physicists, biomedical engineers; nuclear engineers (health physicists) and medical technologists are working in di erent INM all over the country. Nuclear medicine physicians are well trained in the eld of nuclear medicine along with radiation protection. Biomedical engineers and medical technologist are also trained in radiation protection. On the other hand, Medical Physicists are well trained in radiation dosimetry and could contribute to dose assessments, dose measurements, review and validation of dosimetry data. However, there is a need to provide additional training to physician, medical physicists, biomedical engineer, nuclear engineers (health physicists) and medical technologists prior to their involvement in nuclear and radiological emergencies. A formal academic/training course on nuclear or radiological emergency is now o ering by the Department of Nuclear Science and Engineering of Military Institute of Science and Technology (MIST) to the MSc and PhD students.

\section{Conclusion}

The initial response to a radiological emergency may include radiation detection, population monitoring, decontamination, and dose assessment. Therefore, NMPs can play an important role in Case of a Nuclear or Radiological Emergency in Bangladesh. The roles of NMPs should be outlined in the NNERP.

\section{References}

1. Tsubokura M, Gilmour S, Takahashi K, Oikawa T, Kanazawa Y., Internal radiation exposure after the Fukushima nuclear power plant disaster. JAMA. 308: 669-670, 2012.

2. A. S. Mollah, and M. Rahman, National Nuclear or 


\section{Abstract No. OP-8}

\section{Systematic Development of Radiation} Protection in Nuclear Medicine Facilities

${ }_{1}$ Aleya Begum, ${ }_{2}$ M M M Siraz, 2 J. Ferdous, 2 M.S.

Rahman and ${ }_{2} \mathrm{~A}$. Hoque

${ }_{1}$ Physical Science Division, Bangladesh Atomic Energy Commission, Dhaka, Bangladesh

2Health Physics Division, Atomic Energy Centre, Dhaka, Bangladesh

Introduction: Nuclear medicine is a medical specialty involving application of radioactive substances in diagnosis and treatment of disease. Among the most widely used radionuclides in the eld of nuclear medicine, the following should be highlighted: $99 \mathrm{mTc},{ }_{131} \mathrm{I},{ }_{123} \mathrm{I},{ }_{201} \mathrm{Tl}$ and ${ }_{153} \mathrm{Sm}(1)$.The commonly used radioisotopes in nuclear medicine facilities in Bangladesh as well as in different countries are Tc-99m and I-131. The dose assessment of occupational workers in medical practices is mandatory as per requirements of the Nuclear Safety and Radiation Control (NSRC) Rules-1997 and Bangladesh Atomic Energy Regulatory Authority (BAERA) Act-2012 of Bangladesh. This report evaluates the potential risks of internal contamination and occupational radiation doses of nuclear medicine personnel in nuclear medicine facilities in Bangladesh.

Materials and Methods: Radiation protection in nuclear medicine practices is performed by the following monitoring methods: (I) External monitoring by using Harshaw two element TLD-100 (LiF:Mg,Ti) cards for quarterly basis throughout the country and TL chips for extremity and patients dose monitoring. Harshaw TLD reader had been used for reading out the TLD cards. (II) Workplace monitoring by using beta/gamma \& neutron survey meters, (III) Internal monitoring by indirect bioassay method (urine samples of workers who are using unsealed radioactive sources) and collection of air particulates at workplace by suction of indoor air through lter paper using Staplex air sampler and those were measured by High Purity Germanium (HPGe) Detector, (IV) Measurement of Extremity Dose of Workers in Nuclear Medicine Departments using ring dosimeters embedded with TL chips and (V) Arrangement of National Training Courses for occupational workers on radiation protection for dissemination of knowledge by the comprehensive theoretical and practical classes.

Results and Discussion: From 2010 to 2015, the average dose received by doctors, scientists and sta of nuclear medicine facilities in Bangladesh was $0.0844 \mathrm{mSv}, \quad 0.1543 \mathrm{mSv}$ and $0.2718 \mathrm{mSv}$ respectively. The activity concentration of $99 \mathrm{~m} \mathrm{Tc}$ and ${ }_{131} \mathrm{I}$ in urine samples of workers were ranged from 8.58 to $314.92 \mathrm{~Bq} / \mathrm{l}$ and 6.47 to $283.27 \mathrm{~Bq} / \mathrm{l}$ respectively. The e ective dose of $99 \mathrm{~m} \mathrm{Tc}$ were ranged from 0.402 to $14.7 \mu \mathrm{Sv}$ and those of ${ }_{131} \mathrm{I}$ were ranged from 0.12 to $5.58 \mu \mathrm{Sv}$ (2). The activity concentration of artificial radionuclides in air lter were ranged from 0.19 to $60.67 \mathrm{mBq} / \mathrm{m}_{3}$ with average of $6.4 \pm 0.0$ $\mathrm{mBq} / \mathrm{m}_{3}$ for ${ }_{131} \mathrm{I}$; from 0.25 to $40.27 \mathrm{mBq} / \mathrm{m}_{3}$ with average of $4.17 \mathrm{mBq} / \mathrm{m}_{3}$ for $99 \mathrm{mTc}$. The maximum left and right hand finger dose of occupational workers were found to be $47.14 \mathrm{mSv} / \mathrm{yr}$ and $43.28 \mathrm{mSv} / \mathrm{yr}$ respectively for 50 days use of TL ring dosimeters, while mean value of the workers dose of left and right hand finger were found to be $10.71 \mathrm{mSv} / \mathrm{yr}$ and $10.12 \mathrm{mSv} / \mathrm{yr}$ respectively.

Conclusion: Although effective dose of individual worker in nuclear medicine comply with the requirements of NSRC Rules-1997 of Bangladesh, workers should be followed the radiation protection procedures at workplace in order to implement the ALARA concept.

\section{References}

1. B M Dantas, E A Lucena and A L A Dantas. Internal Exposure in Nuclear Medicine: Application of IAEA Criteria to Determine the Need for Internal Monitoring. Brazilian Archives of Biology and Technology 2008;5:103-7,.

2. M J Ferdous, Z Alam, R.K. Khan, S M Iqubal, A Islam, M A 
Abstract No. OP-9

\section{Effect of Positron Range in PET/CT Imaging: Challenges and Solutions}

M M Ahasan, R Khatun, S Akter, M F Uddin, A N Monika, M K Islam, N Sultana

Medical Physics Research Group, BAEC,

\section{Dhaka - 1000 Abstract}

Before undergoing the annihilation process in human tissue, the positron travels a defnite distance. Uncertainty in the localization of the decaying nucleus arises due to the positron range which increases with increasing positron initial energy. The influence of positron range limit to the spatial resolution of PET/CT scanner which blurs the images. This effect is a minor concern in whole body imaging with ${ }_{18} \mathrm{~F}$, but it is a major issue when imaging with higher positron energies isotopes such as ${ }_{13} \mathrm{~N}$ or ${ }_{82} \mathrm{Rb}$. This article provides an overview of current PET/CT scanner technology, a discussion of challenges faced by positron range, and solutions.

\section{Abstract No. OP-10}

\section{Shielding design for unwanted neutron} produced in high energy LINAC

M F Uddin, M S Rahman, M M Ahasan, R

Khatun, S Akter, A N Monika

Medical Physics Research Group, BAEC,

\section{Dhaka - 1000 Abstract}

Neutrons are produced, when linear accelerators is operated at energies above $8 \mathrm{MeV}$, in photo neutron reactions with high $\mathrm{Z}$ materials included in the accelerator head, speci cally in the target, the jaws, the attening lter, and the multi-leaf collimator system . Photo neutron released from linac head, it enters into the body in the form of thermalized neutron. The total neutrons produced are composed of two parts: photonuclear reactions via bremsstrahlung, and electron production via virtual photons. Thus, high-energy x-ray beams (e.g., $>10 \mathrm{MV}$ ) are contaminated with neutrons. The cross sections for $(e, n)$ reactions are smaller by a factor of about 10 than those for $(\gamma, \mathrm{n})$ reactions. These neutrons energies are gradually decreased after multiple scattering from walls, roof, and floor, and consequently, the proportion of the fast neutron (> $0.1 \mathrm{MeV}$ ) reaching inside the maze is usually small. Concrete walls for $\mathrm{x}$-ray shielding purpose are su cient for protection against neutrons. However, the door must be protected against neutrons that diffuse into the maze and reach the door. Reduction in the neutron fluence depends on the accelerator configuration. In apparently ideal case, decrease in neutron fluence of two orders of magnitude (10-2) from machine location to the inside of the maze is expected. Longer maze arrangement i.e., more than $5 \mathrm{~m}$ is desirable in reducing the neutron fluence at the door. Finally, a few inches of a hydrogenous material such as polyethylene is sandwiched between two layers of lead in the door to thermalize the neutrons. First and second lead layers in the door are used to attenuate $\gamma$ ray from consecutive end $\gamma$ reaction.

\section{Abstract No. OP-11 \\ Determination of Normal Ranges of T3, T4, FT4 \& TSH in Healthy Individuals of NINMAS by Using Korea and China Kits.}

Israque Hossain Ansari, Mizanul Hasan, Anwarul-Azim, Haroun-or-Rashid, Sakera Khatun, Zakir Hossain, Mustafa Mamun

\section{National Institute Nuclear Medicine and Allied Sciences}

Objective: The aim of the present study to determine the own normal range of T3, T4, FT3, FT4, \& TSH by two kits, one from Korea and another from China in the laboratory of healthy individuals from 
automated RIA. The China kit was done manually by counting in STRATEC- $\gamma$ Counter of Germany. T3, T4, FT4, \&TSH are done by RIA method and Only TSH by IRMA method by both kits.

Results: The results are analyzed by determining a mean \pm 2 SD for the normal range. This assumes a Gaussian distribution for the analyte and automatically classi es $5 \%$ of the healthy individuals as abnormal. The normal range of T3, T4, FT4 and TSH done by Korean kit were 0.4735932561 $1.8246783301 \mathrm{ng} / \mathrm{ml}$ (Lea et normal range, 0.60 $2.10 \mathrm{ng} / \mathrm{ml}$ ), $62.7115526-159.8398268 \mathrm{ng} / \mathrm{ml}$ (Lea et normal range, $45.00-120.00 \mathrm{ng} / \mathrm{ml}), 7.406228086$ - $21.673082254 \mathrm{ng} / \mathrm{ml}$ (Lea et normal range, 9.01 $23.17 \mathrm{ng} / \mathrm{ml}$ ) and 0.077556152 - 5.462788676 $\mu \mathrm{IU} / \mathrm{ml}$ (Lea et normal range, $0.30-$

$5.00 \mathrm{ng} / \mathrm{ml}$ ). The normal range of T3, T4, FT4, and TSH done by China kit were 0.922455645 $1.929268493 \mathrm{ng} / \mathrm{ml}$ (Lea et normal range, $0.80-2.30$ $\mathrm{ng} / \mathrm{ml}$ ), $44.87278419-141.27125475 \mathrm{ng} / \mathrm{ml}$ (Lea et normal range, 42.00 - $135.00 \mathrm{ng} / \mathrm{ml}), 5.856439522$ $32.703215658 \mathrm{pmol} / \mathrm{L}$ (Lea et normal range, $9.50-$ $25.50 \mathrm{pmol} / \mathrm{L})$ and $0.798176051-5.682858431$ $\mathrm{mIU} / \mathrm{L}$. (Lea et normal range, $0.30-5.00 \mathrm{mIU} / \mathrm{L}$ ).

Conclusion: By estimating few more samples we may use our won estimated normal ranges instead of the lea et normal ranges.

Key Words: In-vitro Lab, RIA, IRMA, Kits, Thyroid and its related Hormones.

\section{Abstract No. OP-12}

\section{Estimating the shielding requirement for a newly planned PET/CT facility}

1Md Nahid Hossain, ${ }_{2}$ Kamila Afroj Quadir, 1Ferdoushi Begum, ${ }_{1}$ Tanvir Ahmed Biman, ${ }_{1} \mathrm{Md}$ Anwar-Ul-Azim, ${ }_{3}$ Rajada Khatun \& 1 Md Nurul Islam ${ }_{1}$ National Institute of Nuclear Medicine \& Allied
Sciences, BSMMU Campus, Shahbag, Dhaka

2Bio- Science Division, Bangladesh Atomic Energy Commission

«Medical Physics Division, Atomic Energy Centre, Dhaka

\section{Abstract}

Positron Emission Tomography (PET) combined with Computed Tomography (CT) is a powerful and very sensitive diagnostic tool that integrates functional and anatomical imaging into one combined scanning system. Positron emission tomography is based on the characteristic way in which positrons annihilate by combining with an electron. This process usually results in the emission of two $511 \mathrm{KeV}$ photons which travel in opposite directions. These $511 \mathrm{KeV}$ annihilation photons are much higher energy than other diagnostic radiations. Because of this high energy of the annihilation radiation, shielding requirements for a PET facility are different from most of the other diagnostic imaging facilities and it's a very important consideration in the design of a PET or PET/CT imaging facility. As a result, significant shielding may be required indoors and ceilings as well as adjacent walls in a PET/CT facility. In this work we present the methods for estimating the shielding requirements for a newly planned PET/CT facility. Shielding calculations of adjacent walls were presented for both controlled and uncontrolled areas. Formulas were used to calculate the shielding materials following the basic AAPM (American Association of Physicists in Medicine) guidelines. This mathematical analysis of the shielding estimation is very useful for a newly planned PET/CT facility. Adequate safe planning with vendor, facility architect and a qualified medical physicist are 